\title{
Estenose arterial subclávia bilateral: mais do que uma descida da tensão arterial - Relato de caso
}

Inês Leite da Silva,* Elsa Martins**

\section{RESUMO}

Introdução: A isquémia crónica dos membros superiores é uma condição rara, sobretudo se bilateral. Dependendo da localização e rede de colaterais, pode permanecer paucissintomática ou cursar com isquémia sintomática dos membros superiores e risco acrescido de eventos coronários e cerebrovasculares. Apesar de o diagnóstico ser imagiológico, o exame objectivo pode revelar alterações, nomeadamente diferenças nos valores tensionais medidos nos dois braços, numa oclusão unilateral. Neste caso clínico, os valores tensionais eram consistentemente baixos bilateralmente, centrando a abordagem inicial na aparente descida tensional.

Descrição do caso: Utente do sexo feminino de 77 anos de idade, hipertensa controlada e com dislipidémia em que, a partir de determinada altura, os valores tensionais medidos na artéria braquial se apresentavam persistentemente inferiores a 90/60 mmHg. A sintomatologia resumia-se a "cansaço" com movimentos amplos dos membros superiores. Na ausência de outras alterações optou-se pela redução subsequente da medicação anti-hipertensora, sem melhoria dos valores tensionais ou da clínica. Após alguns meses, mantendo-se este quadro, foi constatada, no exame objectivo, uma diminuição bilateral dos pulsos radiais e aumento do índice tornozelo-braquial, colocando a hipótese de patologia arterial dos membros superiores, posteriormente confirmada por Eco-doppler arterial que evidenciou oclusão arterial subclávia bilateral.

Comentário: Os valores tensionais medidos na artéria braquial eram aqui erroneamente baixos, dada a oclusão subclávia bilateral salientando-se que, nestes casos, a avaliação tensional baseada numa só medição na artéria braquial pode condicionar uma abordagem errada. O caso evidencia ainda a importância da integração de todos os sinais e sintomas, bem como do exame objectivo no diagnóstico.

Palavras-Chave: Doença Arterial Periférica; Membros Superiores; Avaliação da Tensão Arterial; Hipotensão.

\section{INTRODUÇÃO}

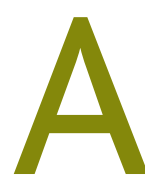
doença vascular periférica, associada à elevada prevalência de doença cardiovascular, é um diagnóstico frequente na prática clínica do médico de família. A avaliação de doença vascular periférica nos membros inferiores acaba por ser realizada de forma mais sistemática. Já a patologia arterial dos membros superiores é menos frequente e por isso menos avaliada. É mais comumente secundá-

* Médica interna de Medicina Geral e Familiar

Unidade de Saúde Familiar Santa Joana - ACeS Baixo Vouga - Aveiro - Portugal

**Assistente de Medicina Geral e Familiar

Unidade de Saúde Familiar Santa Joana - ACeS Baixo Vouga - Aveiro - Portugal ria a oclusão arterial subclávia, sobretudo à esquerda, sendo a oclusão bilateral uma condição rara. ${ }^{1-3}$

Dependendo da localização e da rede de vasos colaterais, pode permanecer paucissintomática ou cursar com isquémia sintomática dos membros superiores (com claudicação ou fadiga muscular, dor em repouso e gangrena digital), bem como risco acrescido de eventos coronários e cerebrovasculares. ${ }^{2-5}$

O diagnóstico definitivo é imagiológico, efectuado por Eco-doppler, mas a suspeição clínica de isquémia dos membros superiores baseia-se na história e no exame objectivo, sendo a manifestação mais comum a diferença de valores tensionais entre os dois membros 
superiores. ${ }^{5-6}$ Uma diferença igual ou superior a 10 $\mathrm{mmHg}$ leva a suspeitar de estenose subclávia unilateral, mesmo em doentes assintomáticos. ${ }^{3-5,7}$ Neste sentido, as orientações actuais recomendam uma avaliação da tensão arterial em ambos os membros superiores numa primeira avaliação, seguida de medição sistemática no membro em que foram detectados valores mais elevados. ${ }^{7-9}$ No presente caso clínico, os valores tensionais eram consistentemente baixos bilateralmente, centrando-se a abordagem inicial na procura de uma etiologia justificando a aparente descida da tensão arterial. Desta forma, um gesto frequente na prática do médico de família como a medição tensional deve ser integrado num exame objectivo atento que, neste caso, incluía ainda a avaliação tensional nos membros inferiores, bem como todos os dados da história clínica.

Assim, o presente caso evidencia uma situação frequente na prática clínica do médico de família, uma descida dos valores tensionais, que acaba por se revelar numa patologia rara: a oclusão arterial subclávia bilateral.

\section{DESCRIÇÃO DO CASO}

O caso reporta-se a uma utente de 77 anos de raça caucasiana, reformada da profissão de enfermeira. No que respeita à caracterização familiar, viúva, pertencendo a uma família unitária. Com antecedentes pessoais de hipertensão controlada com três classes de anti-hipertensores (associação de irbesartan 300mg com hidroclorotiazida $12,5 \mathrm{mg}$, nebivolol $5 \mathrm{mg}$ e indapamida 1,25mg) e dislipidémia medicada com sinvastatina $20 \mathrm{mg}$. Sem antecedentes familiares relevantes.

Tratava-se de uma utente seguida em consulta de hipertensão com a sua médica de família em que, numa destas consultas, a utente referia desde há uma semana valores de tensão arterial sempre inferiores a 100/90 mmHg e "cansaço" com movimentos amplos dos membros superiores, sem outras queixas. Ao exame objectivo, a única alteração eram os valores tensionais $(80 / 50$ $\mathrm{mmHg}$ ). Optou-se por manter a avaliação tensional no ambulatório e ajustar a medicação anti-hipertensora, reduzindo a dose de irbesartan para metade. Contudo, este quadro manteve-se nas semanas seguintes com subsequentes ajustes na medicação anti-hipertensora visando eliminar um potencial efeito iatrogénico na descida dos valores tensionais, terminando medicada com nebivolol 5mg e irbesartan 150mg. Foram, então, realizados exames complementares de diagnóstico (ecocardiograma, electrocardiograma e estudo analítico com hemograma, glicémia, avaliação da função renal e função tiroideia, ionograma, velocidade de sedimentação e proteína $\mathrm{C}$ reactiva). $\mathrm{O}$ estudo analítico e o electrocardiograma não revelaram alterações. $\mathrm{O}$ ecocardiograma apresentava algumas alterações, nomeadamente um alargamento ligeiro da aurícula esquerda e patologia valvular degenerativa (fibroesclerose na válvula aórtica, mantendo normal amplitude de abertura sistólica e válvula mitral com incipiente doença fibrocalcificante a nível do anel, com envolvimento dos folhetos, mantendo normal amplitude de excursão diastólica). Assim, as alterações encontradas nos exames complementares de diagnóstico não justificavam o contexto clínico.

Cerca de dois meses após o início do quadro voltou à consulta, verificando-se ao exame objectivo uma enorme dificuldade em obter valores tensionais nos membros superiores, bem como uma diminuição bilateral da amplitude dos pulsos radiais e um índice tornozelo-braquial (ITB) de 2,7. Tais alterações levantaram a hipótese de patologia arterial dos membros superiores. Discutida esta hipótese de diagnóstico com a doente, com a necessidade de realização de exames complementares de diagnóstico adicionais, esta optou por recorrer a consulta de cirurgia vascular no privado.

Volvidos cinco meses após o início do quadro voltou à consulta com a sua médica de família, com informação clínica do cirurgião vascular e resultado de Eco-doppler arterial dos membros superiores e carotídeo, evidenciando isquémia de todo o território arterial dos membros superiores com oclusão arterial subclávia bilateral, a jusante da emergência das artérias vertebrais, sem mensuração do grau de oclusão. Estava medicada com pentoxifilina $400 \mathrm{mg}$ bid, com indicação para manutenção do controlo clínico com medição dos valores tensionais nos membros inferiores por ser este o valor mais próximo do real. Face à necessidade de esclarecimento etiológico e eventual intervenção terapêutica foi referenciada para a consulta de cirurgia vascular do hospital de referência.

Um ano depois teve alta desta consulta, trazendo informação clínica daquele seguimento, confirmando o 
diagnóstico de oclusão arterial subclávia bilateral. Cerca de um ano após o episódio agudo encontrava-se com boa revascularização e paucissintomática, ficando em aberto a etiopatologia: degenerativa em consonância com a idade e ateromatose ou dissecção crónica no contexto de hipertensão? O esclarecimento etiológico exigiria exames angiográficos invasivos cujo risco ultrapassaria eventuais benefícios, dado não existir indicação para tratamento invasivo face ao quadro paucissintomático, idade da doente e risco inerente à correcção anatómica, optando-se pelo tratamento conservador. Assim, face ao risco cardiovascular global muito alto, tratando-se de uma doente hipertensa com doença arterial periférica, foi delineado um plano para prevenção secundária. Este plano passou por:

- Associação de ácido acetilsalicílico 150mg id;

- Optimização do controlo tensional com novo ajuste gradual da medicação anti-hipertensora, acabando por se reintroduzir a associação de irbesartan $300 \mathrm{mg}$ com hidroclorotiazida $12,5 \mathrm{mg}$ e mantendo nebivolol 5mg;

- Relativamente ao controlo lipídico apresentava um valor de colesterol LDL de 66mg/dl, pelo que se manteve a terapêutica com sinvastatina $20 \mathrm{mg}$;

- Discussão de possíveis modificações no estilo de vida, nomeadamente pela prática regular e continuada de exercício físico (optando pela realização de caminhadas diárias de cerca de 30 minutos), bem como pela adopção de uma dieta variada, nutricionalmente equilibrada, pobre em gorduras e enriquecida em fibras, com aumento do consumo de fruta, vegetais, legumes, nozes, peixe, cereais e pão integrais.

Actualmente, a doente mantém-se paucissintomática sem limitação nas suas actividades de vida diária, mantendo vigilância com medição dos valores tensionais nos membros inferiores, estando medicada com pentoxifilina 400mg bid, ácido acetilsalisílico 150mg id, sinvastatina $20 \mathrm{mg}$ id e terapêutica anti-hipertensora entretanto optimizada, com bom controlo tensional e dos valores da ficha lipídica.

\section{COMENTÁRIO}

Numa primeira abordagem, o quadro clínico simulava um caso de hipertensão em que subitamente os valores tensionais se tornaram inferiores a $90 / 60 \mathrm{mmHg}$, de forma persistente, sem relação com o ortostatismo ou as refeições e sem sintomas de hipotensão. Não existe um valor consensual a partir do qual se possa considerar a tensão arterial "demasiado baixa”. Alguns autores referem, como limiar, valores inferiores a 90/60 mmHg. ${ }^{3}$ A American Heart Association define "hipotensão" pela existência de sintomas associados ou descida abrupta dos valores tensionais (superior a 20 mmHg). ${ }^{10}$

Neste caso clínico, face à descida mantida dos valores tensionais, excluídas outras causas identificáveis para esta, foi-se reduzindo a medicação anti-hipertensora procurando debelar um eventual efeito iatrogénico, sem sucesso.

Um exame objectivo mais atento acabou por ser a chave para a suspeição do diagnóstico, revelando diminuição da amplitude dos pulsos radiais e dificuldade na medição dos valores tensionais na artéria braquial bilateralmente, mantendo-se estes valores elevados nos membros inferiores com um índice tornozelo-braquial elevado. Tais achados orientaram o raciocínio para a hipótese de patologia arterial dos membros superiores. Havia ainda outros dados a favor na história clínica: factores de risco cardiovascular (idade, dislipidémia, hipertensão) e sintomatologia sugestiva de claudicação nos membros superiores (definida pela utente como "cansaço" com a mobilização ampla destes).

Assim, a aparente diminuição dos valores tensionais era afinal uma diminuição do fluxo sanguíneo nos membros superiores por oclusão arterial subclávia bilateral. A avaliação inicial da tensão arterial, segundo as orientações actuais, deve ser efectuada em ambos os membros superiores para identificar eventuais diferenças devidas a doença vascular periférica para, nesse caso, utilizar como valor de referência o mais elevado. Tal aplica-se num caso de oclusão unilateral; ${ }^{7-9}$ neste caso, sendo a oclusão bilateral era igualmente insuficiente. Os valores tensionais medidos nos membros inferiores seriam os mais próximos dos reais valores sistémicos que, aliás, se mantinham elevados.

A principal etiologia da estenose arterial subclávia é aterosclerótica. Outras causas incluem arterite, radiação, síndromas de compressão, displasia fibromuscular e neurofibromatose. ${ }^{2}$ Relativamente à abordagem, ainda pouco se conhece acerca da história natural da estenose subclávia, mas o prognóstico é geralmente be- 
nigno. Assim, doentes assintomáticos não têm indicação para tratamento invasivo. ${ }^{5}$ Contudo, qualquer oclusão arterial subclávia sintomática deve ser investigada e tratada. O controlo dos factores de risco aterosclerótico deve sempre fazer parte desta abordagem, mesmo em doentes assintomáticos. ${ }^{5-6}$

Concluindo, o presente caso alerta para o diagnóstico de uma condição pouco frequente: a doença arterial dos membros superiores. A questão inicial foi interpretar a descida dos valores tensionais medidos na artéria braquial, salientando que nestes casos a prática generalizada de avaliar a tensão arterial com base numa única medição na artéria braquial pode condicionar abordagens incorrectas.

Sublinha-se, ainda, a importância do exame objectivo no diagnóstico, aliado à valorização do quadro clínico global, bem como a um elevado índice de suspeição, especialmente importante em patologias menos frequentes como a que aqui se apresenta. Em síntese, salienta-se aquele que é o nosso maior desafio: procurar as causas subjacentes a um determinado quadro clínico ao invés de cair na tentação de considerar e tratar queixas isoladas, como uma aparente "descida da tensão arterial”, para evitar a protelação do diagnóstico e a iatrogenia médica e psicológica de exames ou atitudes terapêuticas desnecessárias.

\section{AGRADECIMENTOS}

As autoras agradecem o contributo de Ivo Reis (interno de Medicina Geral e Familiar na USF BRIOSA), Carla Bastos (Médica de Família na USF Alpha) e Yolanda Oliveira (interna de Medicina Geral e Familiar na USF Santa Joana) pelo seu contributo na revisão crítica do artigo.

\section{REFERÊNCIAS BIBLIOGRÁFICAS}

1. Jahangiri B, Al-Shammeri $O$, Veinot JP, Matzinger F, Haddad H. An unusual case of hypertensive diastolic heart failure with bilateral subclavian artery stenosis. Can J Cardiol. 2009;25(2):111-2.

2. Ochoa $\bigvee M$, Yeghiazarians Y. Subclavian artery stenosis: a review for the vascular medicine practitioner. Vasc Med. 2011;16(1):29-34.

3. Shadman R, Criqui MH, Bundens WP, Fronek A, Denenberg JO, Gamst
AC, et al. Subclavian artery stenosis: prevalence, risk factors, and association with cardiovascular diseases. J Am Coll Cardiol. 2004;44(3):61823.

4. McDermot MM. The magnitude of the problem of peripheral arterial disease: epidemiology and clinical significance. Cleve Clin J Med. 2006;73 Suppl 4:S2-7.

5. Mohler III ER. Overview of upper extremity peripheral artery disease. 2013 Aug 21 [revised 2014 Aug; cited 2014 Jun 28]. In UpToDate. Wolters Kluwer; 2014. Available from: http://www.uptodate.com/online

6. European Stroke Organisation, Tendera M, Aboyans V, Bartelink ML, Baumgartner I, Clément D, et al. ESC Guidelines on the diagnosis and treatment of peripheral artery diseases: document covering atherosclerotic disease of extracranial carotid and vertebral, mesenteric, renal, upper and lower extremity arteries: the Task Force on the Diagnosis and Treatment of Peripheral Artery Diseases of the European Society of Cardiology (ESC). Eur Heart J. 2011;32(22):2851-906.

7. Pickering TG, Hall JE, Appel LJ, Falkner BE, Graves J, Hill MN et al. Recommendations for blood pressure measurement in humans and experimental animals - Part 1: blood pressure measurement in humans: a statement for professionals from the Subcommittee of Professional and Public Education of the American Heart Association Council on High Blood Pressure Research. Circulation. 2005;111(5):697-716.

8. Mancia G, Laurent S, Agabiti-Rosei E, Ambrosioni E, Burnier M, Caulfield MJ, et al. Reappraisal of European guidelines on hypertension management: a European Society of Hypertension Task Force document. J Hypertens. 2009;27(11):2121-58.

9. Direcção-Geral da Saúde. Hipertensão arterial: definição e classificação - Norma da Direcção-Geral da Saúde n 20/2011, de 28/09/2011 (actualizada em 19/03/2013). Lisboa: DGS; 2013. Portuguese

10. American Heart Association. Your high blood pressure questions answered: low blood pressure. AHA; 2014 [revised 2014 Apr 8; cited 2014 Mar 10]. Available from: http://www.heart.org/HEARTORG/Conditions/HighBloodPressure/AboutHighBloodPressure/Low-Blood-Pressure_UCM_301785_Article.jsp

\section{CONFLITOS DE INTERESSE}

As autoras declaram não ter conflito de interesses.

\section{ENDEREÇO PARA CORRESPONDÊNCIA}

Inês Alves Leal Leite da Silva

Rua do Lila n. ${ }^{\circ} 5$ AB, 3810-427 Aveiro

E-mail: ines.leite.silva@gmail.com

Recebido em 04-07-2014

Aceite para publicação em 05-09-2014 


\section{ABSTRACT}

MORE THAN JUST A FALL IN BLOOD PRESSURE - A CASE REPORT OF BILATERAL SUBCLAVIAN ARTERY STENOSIS Introduction: Chronic ischaemia of the upper extremities is rare, particularly when it is bilateral. Depending on the existence and location of collateral vessels, it may remain asymptomatic or it may present with symptomatic ischemia of the upper limbs and a high risk of coronary and cerebrovascular disease. Although the diagnosis is made by ultrasonography, the physical examination may reveal abnormalities, including differences in blood pressure values between both arms with unilateral occlusion. In this case report, blood pressure values were consistently low bilaterally, which led to an erroneous initial approach based on a presumed decrease in systemic blood pressure.

Case description: The authors describe the case of a 77 year old female patient, with controlled hypertension and dyslipidaemia, who presented with brachial blood pressure (BP) measurements consistently lower than $90 / 60 \mathrm{mmHg}$, as well as complaints of fatigue with movements of the upper extremities. In the absence of other abnormalities, changes were made to the dose of her anti-hypertensive medication, without clinical improvement. After a few months without change in the clinical situation, the physical examination revealed a bilateral decrease in radial pulses and an increase in the ankle-brachial index, suggesting occlusive upper extremity arterial disease. This was confirmed by an arterial Doppler ultrasound, which revealed bilateral occlusion of the subclavian arteries.

Comment: Blood pressure measurements were erroneously low because they were made at the brachial artery in a patient with a bilateral occlusion of the subclavian arteries. In these cases, the evaluation of BP based solely on brachial measurements may lead to an incorrect approach. It is important to integrate other signs and symptoms as well as a more complete physical examination when evaluating a possible diagnosis of this condition.

Keywords: Peripheral Arterial Disease; Upper Extremity; Blood Pressure Determination; Hypotension. 